\title{
Medidas de Tromboprofilaxis en Artroplastia Total de Rodilla. Práctica habitual en la Comunidad Valenciana y revisión bibliográfica.
}

\author{
DOI: http://dx.doi.org//10.37315/SOTOCAV20202815530
}

\begin{abstract}
ÁLVAREZ-LLANAS $\mathrm{A}^{, 1,2}$, MIFSUT-MIEDES $\mathrm{D}^{1}$, CLIMENT-PERIS $\mathrm{V}^{1}$, BAEZA-OLIETE $\mathrm{J}^{1}$, GARGALLO-VERGE EJ ${ }^{1}$, STRAUCH-LEIRA $\mathrm{M}^{1}$, MARTÍNEZ-ALGARRA JC ${ }^{1}$, VALERO-QUERALT MA ${ }^{1}$, FERRARO-ESPARZA $L^{1}$, GILABERT-DAPENA ${ }^{1}$.
\end{abstract}

1. GRUPO DE ARTROPLASTIA DE RODILLA DE LA COMUNIDAD DE VALENCIANA.

2. HOSPITAL ARNAU DE VILANOVA-LLIRIA VALENCIA.

\section{Resumen.}

Antecedentes: El tromboembolismo es una complicación de la cirugía de artroplastia total de rodilla. Para su prevención disponemos de múltiples medidas físicas y farmacológicas. Objetivo: Conocer qué medidas de prevención tromboembólica son las empleadas por los cirujanos ortopédicos en cirugía protésica de rodilla primaria en diferentes centros hospitalarios de nuestra región. Método: Estudio transversal descriptivo observacional basado en encuesta dirigida a especialistas COT de 9 hospitales públicos de la Comunidad Valenciana y búsqueda bibliográfica. Resultados: Se obtuvieron 64 encuestas. Todos los cirujanos eligen HBPM durante un mes como medida de tromboprofilaxis, descartando anticoagulantes orales o aspirina. El $29 \%$ también emplea dispositivos de movilización pasiva. Conclusiones: Los cirujanos ortopédicos de la comunidad valenciana optan por HBPM conforme a las mejores evidencias. Se estima, que la incidencia de eventos tromboembólicos sintomáticos desciende del 4,3 \% al 1,8\% con el uso de HBPM. El empleo de dispositivos de movilización pasiva y medias de compresión no están avaladas por las evidencias. La estratificación preoperatoria del riesgo de tromboembolismo, para emplear ácido acetil salicílico asociado a bombas de presión intermitente en caso de no existir alto riesgo es una tendencia cada vez más aceptada internacionalmente.

PALABRAS CLAVE: Total knee arthroplasty, thromboprophylaxis.

\section{Summary.}

Background: Thromboembolism is one of the Total Knee Arthroplasty risks. For its prevention we have multiple physical and pharmacological measures availables. Objective: To know which thromboembolic prevention measures are those used by orthopedic surgeons in knee replacement surgery in different hospital centers in our region. Method: Observational descriptive cross-sectional study based on a survey directed at orthopedic surgeons from 9 public hospitals in the Valencian Community and a bibliographic search. Results: 64 completed surveys were obtained. All surgeons choose LMWH for one month as a thromboprophylaxis measure, ruling out oral anticoagulants or aspirin. $29 \%$ also employ passive motion devices. Conclusions: Orthopedic surgeons from the Valencian Community opt for LMWH guided by the best evidence. It is estimated that the incidence of symptomatic thromboembolic events drops from $4.3 \%$ to $1.8 \%$ with the use of LMWH. The use of passive motion devices and graduated compression stockings are not supported by the evidence. The preoperative stratification of the risk of thromboembolism, to use aspirin associated with intermittent pneumatic compression device in the absence of high risk, is an increasingly internationally accepted trend.

\section{Correspondencia:}

Alejandro Álvarez Llanas Hospital Arnau de Vilanova

C/ San Clemente 12.

46015 Valencia.

e-mail: alvarezllanas@yahoo.es 


\section{Introducción}

La artroplastia total de rodilla (ATR) es una cirugía frecuente con un notable éxito. Su indicación principal es el alivio del dolor en la gonartrosis severa. La cirugía no está exenta de riesgos, estando considerado el tromboembolismo uno de los más frecuentes. El tromboembolismo puede ser subclínico o bien manifestarse en forma de dos complicaciones, la trombosis venosa profunda (TVP) y el tromboembolismo pulmonar (TEP).

La TVP sintomática se manifiesta habitualmente por hinchazón, calor y dolor en el miembro inferior afectado. La venografía y la ecografía (dópler o dúplex) permiten detectar las presentaciones sintomáticas y asintomáticas. La venografía es la prueba más sensible ${ }^{1}$. EI TEP se manifiesta en forma de disnea y dolor torácico. Su incidencia es muy inferior a la TVP. En un estudio de 1989 sobre 312 ATR a los que no se les aplico medidas de tromboprofilaxis, la incidencia de TVP medida con venografía fue del $56 \%$ y de TEP sintomático del $1,9 \%{ }^{2}$.

Con independencia de la toma de medidas de profilaxis, la incidencia del tromboembolismo ha disminuido con el paso de los años de forma muy relevante ${ }^{3,4}$. Estudios de incidencia de tromboembolismo sintomático en los años 80 reflejan cifras superiores al $30 \%$. Sin embargo, en la actualidad la estimación de tromboembolismo sintomático prescindiendo de medidas de profilaxis se estima en un $4,3 \%{ }^{5}$. Esta drástica reducción en la incidencia es atribuida a los avances en las técnicas quirúrgica y anestésica, un mejor manejo del dolor y a la incentivación de la deambulación y carga precoz. La estancia media de los pacientes operados ha pasado de 35 días en los años 60 a apenas 4 días en la actualidad. Los datos de incidencia de tromboembolismo de estudios anteriores al año 2000 no deberían ser tenidos en cuenta a lo hora de emplear medidas de profilaxis, pues sobrevaloran la magnitud del problema $^{5}$. Las medidas de profilaxis añaden nuevos riesgos a los propios de la cirugía, como lo es, el aumento del sangrado. La balanza riesgo-beneficio se mueve actualmente en rangos muy estrechos. Algunos autores nos advierten que actualmente la tromboprofilaxis puede generar más daño que beneficio y sus indicaciones deben ser revaluadas ${ }^{6}$.

Para prevenir el tromboembolismo podemos emplear diferentes opciones, que dividimos en medidas físicas y medidas farmacológicas. Son medidas físicas: los dispositivos de movilización pasiva postoperatoria también conocidos como artromotor, las medias de compresión y las bombas de presión intermitente. Lo son farmacológicas: las heparinas de bajo peso molecular (HBPM), los anticoagulantes orales (ACO) y el ácido acetil salicílico (AAS o aspirina) por su efecto antiagregante plaquetario. Medidas físicas y farmacológicas pueden ser empleadas de forma conjunta para mejorar su rendimiento.

El objetivo principal del presente estudio es conocer cuáles de estas medidas de profilaxis tras la ATR primaria son las preferidas por la mayoría de los cirujanos ortopédicos en la Comunidad Valenciana. De forma secundaria realizamos una revisión bibliográfica para conocer el estado del conocimiento actual.

\section{Material y Métodos}

Estudio transversal descriptivo observacional basado en encuesta anónima dirigida a facultativos especialistas en Cirugía Ortopédica y Traumatología de 9 hospitales públicos de la Comunidad Valenciana. Hospital General de Valencia, Hospital de Vinaroz, Hospital de La Ribera (Alcira), Hospital Francesc de Borja de Gandía, Hospital General de Requena, Hospital Clínico-Malvarrosa de Valencia, Hospital de Manises, Hospital La Fe de Valencia, Hospital Arnau de Vilanova-Liria. En el cuestionario se exploran diferentes aspectos del manejo de la cirugía de recambio articular de rodilla y de forma específica sobre las medidas de profilaxis habitualmente empleadas (Fig. 1): medias de compresión, bomba de compresión intermitente, movilización pasiva postoperatoria y elección y duración de profilaxis farmacológica. Además, se preguntó sobre la categoría de su hospital y cantidad de prótesis que implanta ajustado en 3 rangos, menos de 10, entre 10 y 50 o más de 50 prótesis al año.

La revisión bibliográfica y actualización sobre la profilaxis antitrombótica se realizó mediante búsqueda en PubMed en diciembre de 2019. Los términos de búsqueda empleados fueron: "knee arthroplasty or TKA", "prophylaxis or thromboprophylaxis or thromboembolism", "passive motion", "graduated compression stockings", "intermittent pneumatic compression or mechanical compression", "aspirin".

Se revisaron según criterios de calidad las principales guías de práctica clínica, los ensayos clínicos controlados aleatorizados, las revisiones sistemáticas y los metaanálisis.

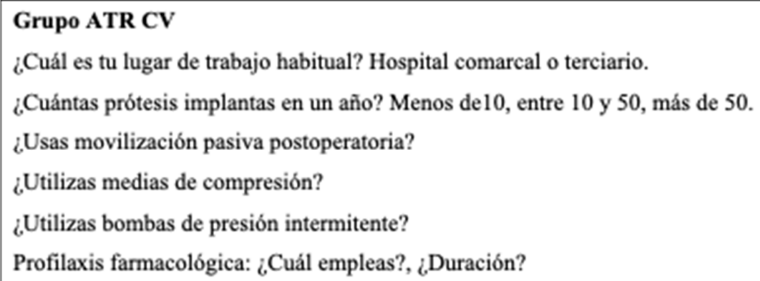

Figura 1. Preguntas incluidas en el cuestionario del Grupo ATR CV.

\section{Resultados}

Se obtuvieron un total de 64 encuestas correctamente contestadas. Los cirujanos ortopédicos consultados pertenecen en un $64 \%(41 / 64)$ a hospitales comarcales y un $36 \%(23 / 64)$ a hospitales terciarios. El $89 \%(57 / 64)$ de los cirujanos implantan entre 10 y 50 prótesis al año, $4,7 \%$ (3/64) más de 50 y un 6,3\% (4/64) declararon poner menos de 10 al año. 
Todos los cirujanos encuestados emplean HBPM como medida de tromboprofilaxis farmacológica. Un tercio de ellos emplean además dispositivos de movilización pasiva en el postoperatorio. Un encuestado emplea medias de compresión. Las bombas de presión intermitente no son empleadas por ningún cirujano (Tabla I) (Fig. 2).

\begin{tabular}{|l|c|}
\hline \multicolumn{2}{|l|}{$\begin{array}{l}\text { Medidas de profilaxis tromboembólica } \\
\text { empleadas }\end{array}$} \\
\hline Total, encuestados & 64 \\
\hline $\begin{array}{l}\text { Movilización pasiva } \\
\text { postoperatoria }\end{array}$ & $19(29 \%)$ \\
\hline Medias de compresión & $1(1,5 \%)$ \\
\hline $\begin{array}{l}\text { Bomba de compresión } \\
\text { intermitente (BPI) }\end{array}$ & $0(0 \%)$ \\
\hline $\begin{array}{l}\text { Heparina bajo peso } \\
\text { molecular (HBPM) }\end{array}$ & $64(100 \%)$ \\
\hline
\end{tabular}

Tabla I. Medidas de tromboprofilaxis empleadas según resultado de la encuesta.

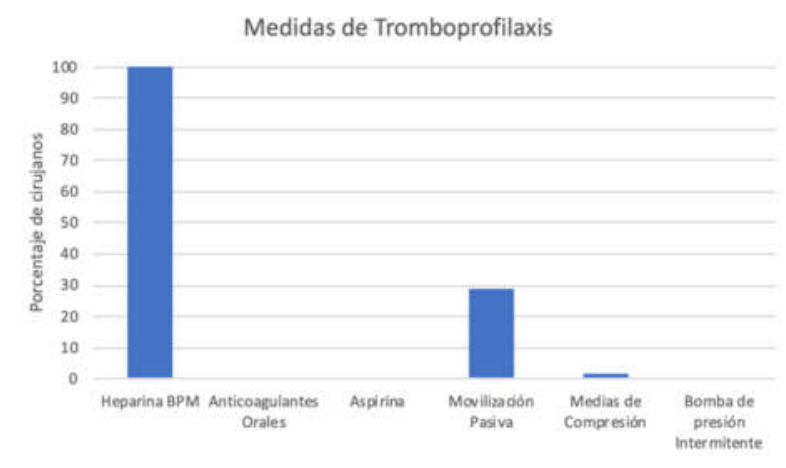

Figura 2. Medidas de tromboprofilaxis empleadas tras la artroplastia primaria de rodilla.

\section{Discusión}

La novena edición de la American College of Chest Physicians (ACCP) ${ }^{7}$, también conocida como "CHEST 9", es considerada sin duda la "Biblia" de las guías clínicas en tromboprofilaxis. Dirigida por prestigiosos especialistas en medicina basada en la evidencia, su rigor metodológico en el análisis de las pruebas, alejado de los conflictos de interés y planteando un abordaje centrado en el paciente, la ha convertido en la mejor guía para la mayoría de los autores ${ }^{8}$. Supuso un importante cambio respecto a guías anteriores. Nosotros nos hemos basado en las recomendaciones de esta guía y la de la American Academy of Orthopaedic Surgeons $(\mathrm{AAOS})^{9}$ para valorar los resultados de la encuesta, incorporando a la discusión los ensayos clínicos más relevantes de nuestra búsqueda en Pubmed. La guía de tromboprofilaxis de la SECOT se encuentra actualmente en revisión.

Profilaxis farmacológica

La guía de la American College of Chest Physicians (ACCP) recomienda las HBPM por encima del resto de opciones de quimioprofilaxis. De acuerdo con esta guía, todos los cirujanos ortopédicos de nuestra encuesta utilizan HBPM como principal forma de profilaxis tromboembólica. Según esta guía la warfarina (Aldocumar $\AA$ ), fondaparinux (Arixtra ${ }^{\circledR}$ ) y rivaroxaban (Xarelto $®$ ) han demostrado similar capacidad preventiva que las HBPM, pero con un mayor número de complicaciones por sangrado. Por su parte el dabigatrán (Pradaxa $®$ ) y apixaban (Eliquis $®$ ) son similares a la HBPM en términos de eficacia y propensión a causar hemorragias, según pruebas de calidad moderada. Sin embargo, una mayor experiencia a largo plazo con HBPM todavía favorece su uso. La aspirina ha demostrado ser tan segura como la HBPM, pero no tan eficaz en evitar eventos tromboembólicos asintomáticos. Estudios indirectos ofrecen mayor eficacia a las $\mathrm{HBPM}^{5}$.

Los cirujanos ortopédicos encuestados prolongan el uso de la HBPM durante un mes. La guía CHEST 9 recomienda prolongar el tratamiento con HBPM hasta 35 días después de la intervención. Según sus propias estimaciones si no se emplea profilaxis el riesgo de tromboembolismo sintomático en los primeros 14 días es del 2,8\%, y del día 15 al 35 del 1,5\%. Si empleamos HBPM el riesgo en los primeros 14 días disminuye a más de la mitad quedando en un $1,15 \%$ y en la segunda quincena $0,65 \%$. El riego de tromboembolismo el primer mes sin profilaxis es de $4,3 \%(2,8 \%$ de TVP y $1,5 \%$ de TEP) y con HBPM de $1,8 \%(1,25 \%$ TVP, $0,55 \%$ TEP $)$.

Asociación Ácido Acetil Salicílico - Bombas de Presión Intermitente

Tanto la guía de la American College of Chest Physicians (ACCP) como la de la American Academy of Orthopaedic Surgeons (AAOS) incluyen a la aspirina como una posibilidad de tromboprofilaxis. Su marcado menor coste y su cómoda administración oral la convierten en una opción muy atractiva. Desde la publicación de estas guías en 2012 el interés por la aspirina ha aumentado y se han publicado varias investigaciones asociando su uso a las bombas de presión intermitente con resultados favorables. Un ensayo clínico aleatorizado realizado en 2014 en 120 pacientes no haya diferencias entre los grupos HBPM más profilaxis mecánicas y aspirina más profilaxis mecánica ${ }^{10}$. Un reciente estudio observacional publicado en 2019 en la revista JAMA por Hood y colaboradores incluye 41.537 pacientes $^{11}$. En él se hace un análisis de no inferioridad de la aspirina frente a un grupo anticoagulado (con HBPM o ACO) y un grupo 
sin quimioprofilaxis. Los eventos tromboembólicos aparecieron en el $1,1 \%$ de los tratados solo con aspirina, $1,4 \%$ de los anticoagulados y $4,8 \%$ de los que no recibieron quimioprofilaxis. En la mayoría de los pacientes se asoció bombas de presión intermitente, ácido tranexámico, anestesia regional y movilización precoz. La aspirina mostro no ser inferior al resto de anticoagulantes y una opción válida en la prevención del tromboembolismo. El estudio tiene sesgos, no es aleatorizado y el cirujano responsable decide qué medida de profilaxis emplear según sus creencias, pero el tamaño muestral lo convierte en un trabajo de resultados relevantes y que pone de manifiesto la extensión del uso de aspirina en los Estados Unidos ${ }^{11,12}$ En Europa la European Society of Anaestehesiology publica una guía en $2018^{13}$ que recomienda la aspirina como opción de prevención del tromboembolismo en artroplastia total de rodilla y cadera. Sugiere emplearla cuando el paciente no tenga un alto riesgo de trombosis venosa y asociarlo al empleo de bombas de presión intermitente.

\section{Profilaxis Mecánica}

Los sistemas mecánicos de profilaxis tienen la ventaja de poder disminuir el tromboembolismo sin aumentar el riesgo de sangrado. Dado que en la actualidad la incidencia de tromboembolismo sintomático tras la ATR ha disminuido gracias a la movilización precoz y las mejoras en la técnica quirúrgica y anestésica, algunos autores abogan por emplear solo medidas de profilaxis mecánicas y en el caso de presentar síntomas administrar el tratamiento con anticoagulantes ${ }^{14,15}$.

\section{Bombas de presión intermitente}

Los ensayos clínicos con bombas de presión intermitente (BPI) o dispositivos de presión neumática intermitente, han demostrado que son una medida de profilaxis tromboembólica eficaz, pero por si solas inferiores a la quimioprofilaxis ${ }^{16,17}$. Presentan como principal ventaja un menor sangrado postoperatorio ${ }^{18}$. El empleo combinado de BPI con HBPM potencian sus efectos preventivos ${ }^{19-21}$. La guía Chest 9 de la ACCP recomienda emplear BPI más HBPM en pacientes con riesgo elevado de tromboembolismo ${ }^{7}$. También se ha demostrado su efecto sinérgico asociado con aspirina, llegando a una incidencia del $0 \%$ de tromboembolismo subclínico en pacientes catalogados de bajo riesgo tromboembólico ${ }^{22}$. Pese a ser una opción de prevención de tromboprofilaxis eficaz, ninguno de nuestros encuestados reconoció su uso. El inconveniente de las BPI es su aparatosidad y además para que su uso sea efectivo se recomienda emplearse al menos 18 horas al día $^{7}$ y prolongarse mientras haya riesgo. La duración óptima para esta profilaxis mecánica es desconocida ${ }^{23}$. Algunos autores prescriben su uso solo durante el ingreso y otros lo prolongan hasta 6 semanas después del alta hospitalaria. Para estos supuestos se han creado dispositivos portátiles más manejables.

\section{Dispositivos de movilización pasiva postoperatoria}

Se ha atribuido a los dispositivos de movilización pasiva postoperatoria la capacidad de disminuir los eventos tromboembólicos ${ }^{24}$. Un $29 \%$ de los cirujanos encuestados emplean dispositivos de movilización pasiva en el postoperatorio. Sin embargo la utilidad de estos dispositivos no parece estar sustentado por las evidencias. En 2014 una revisión sistemática y metaanálisis Cochrane sobre la eficacia de estos dispositivos en la prevención del tromboembolismo tras ATR concluye que no existen pruebas de su utilidad. De un total de 405 pacientes incluidos en el metaanálisis un $18 \%$ del grupo de movilización pasiva desarrolla TVP, frente solo un $15 \%$ del grupo control, aunque estas diferencias no fueron estadísticamente significativas ${ }^{25}$. En 2015 el Hospital for special surgery de Nueva York, considerado el primer hospital de Estados Unidos en cirugía ortopédica, publicó un ensayo prospectivo aleatorizado en el que no encontraron ningún beneficio en prevención de tromboembolismo, rango de movilidad o acortamiento de estancia y si un elevado coste. Estos dispositivos dejaron de ser empleados en su institución a raíz de los resultados obtenidos ${ }^{26}$. La guía de la ACCP CHEST 9 no contempla el empleo de dispositivos de movilización pasiva postoperatoria como medida de tromboprofilaxis.

\section{Medias de compresión}

La guía CHEST 9 no contempla el empleo de medias de compresión como medida de tromboprofilaxis. Solo uno de nuestros encuestados se inclina por su uso. Los estudios científicos hallados en nuestra revisión, no avalan el empleo de las medias de compresión en la prevención del tromboembolismo. Un ensayo clínico de 1995 sobre 177 pacientes no halló diferencias estadísticamente significativas en el desarrollo de tromboembolismo entre el grupo de prevención con medias de compresión y el grupo control sin profilaxis ${ }^{27}$. Un ensayo clínico publicado en 2009, aleatorizó a 440 pacientes con bajo riesgo de tromboembolismo en 4 grupos: sin profilaxis, medias de compresión, bomba de presión intermitente y HBPM. La incidencia de tromboembolismo medido con eco duplex fue la misma entre el grupo control y el grupo con medias de compresión (22\%) y en ambos grupos un paciente desarrollo TEP. Los grupos, bomba de presión intermitente $(8 \%)$ y de HBPM $(6 \%)$ si presentaron un descenso significativo del tromboembolismo ${ }^{18}$. Un trabajo de 2014 compara la asociación de medias de compresión y HBPM frente a un grupo que emplea bombas de presión intermitente y HBPM. En el grupo de medias de compresión la incidencia del tromboembolismo medido con ecografía fue del $40 \%$ frente a un $0 \%$ en la combinación HBPM y $\mathrm{BPI}^{19}$. Un trabajo publicado en 2016 sobre 201 pacientes compara el uso de medias de compresión más HBPM frente a solo HBPM no encontrando diferencias estadísticamente significativas en la detección o desarrollo de tromboembolismo ${ }^{28}$. 


\section{Conclusiones}

Todos los cirujanos ortopédicos de la Comunidad Valenciana optan por las HBPM y prolongan su prescripción durante un mes conforme a las mejores evidencias. Se estima, que la incidencia de eventos tromboembólicos sintomáticos desciende del 4,3\% al $1,8 \%$ gracias al empleo de las HBPM. Un $29 \%$ de los encuestados emplean dispositivos de movilización pasiva postoperatoria, no estando su uso avalado por las evidencias.
El empleo de medias de compresión no previene el tromboembolismo tras la ATR. Las medidas de tromboprofilaxis pueden generar más daño que beneficio y sus indicaciones están siendo revaluadas.

La estratificación preoperatoria del riesgo de tromboembolismo, para emplear aspirina asociada a las bombas de presión intermitente en caso de no existir alto riesgo es una tendencia cada vez más aceptada internacionalmente. 


\section{Bibliografía}

1. Chang MJ, Song MK, Kyung MG, Shin JH, Chang CB, Kang SB. Incidence of deep vein thrombosis before and after total knee arthroplasty without pharmacologic prophylaxis: a 128-row multidetector CT indirect venography study. BMC Musculoskelet Disord 2018; 19(1):274. doi: 10.1186/s12891-018-2166-8. PMID:30064411.

2. Stringer MD, Steadman CA, Hedges AR, Thomas EM, Morley TR, Kakkar VV. Deep vein thrombosis after elective knee surgery. An incidence study in 312 patients. The Journal of Bone and Joint Surgery. British Volume 1989; 71(3):492-7.

3. Warren JA, Sundaram K, Anis HK, Kamath AF, Higuera CA, Piuzzi NS. Have Venous Thromboembolism Rates Decreased in Total Hip and Knee Arthroplasty? J Arthroplasty 2020; 35(1):259-264. doi: 10.1016/j.arth.2019.08.049. Epub 2019 Aug 29. PMID:31530463.

4. Bjørnarå BT, Gudmundsen TE, Dahl OE. Frequency and timing of clinical venous thromboembolism after major joint surgery. J Bone Joint Surg Br 2006; 88(3):386-391. [PubMed] [Google Scholar]

5. Falck-Ytter Y, Francis C W, Johanson N A, Curley C, Dahl O E, Schulman S, Ortel T L, Pauker S G, Colwell C W. Jr. Prevention of VTE in orthopedic surgery patients: Antithrombotic therapy and prevention of thrombosis, 9th ed: American College of Chest Physicians Evidence-Based Clinical Practice Guidelines. Chest 2012; 141 (2, Supplement): e278S-e325S.

6. Kotaska A. Venous thromboembolism prophylaxis may cause more harm than benefit: an evidence-based analysis of Canadian and international guidelines. Thromb J 2018;16:25. doi: 10.1186/s12959-018-0180-6. PMID: 30337840; PMCID: PMC6178253.

7. Guyatt GH, Norris SL, Schulman S, Hirsh J, Eckman MH, Akl EA, et al. Methodology for the development of antithrombotic therapy and prevention of thrombosis guidelines: Antithrombo- tic Therapy and Prevention of Thrombosis, 9th ed: American College of Chest Physicians Evidence-Based Clinical Practice Guidelines. Chest 2012; 141 Suppl. 2:S53-70.

8. Ruiz-Iban MA, Díaz-Heredia J, Elías-Martín ME, Martos-Rodríguez LA, Cebreiro-Martínez del Val I, PascualMartín-Gamero FJ. Las nuevas guías de profilaxis de enfermedad tromboembólica venosa en artroplastia de cadera y rodilla electivas: ¿nos acercamos o nos alejamos del consenso? [The new guidelines for deep venous thromboembolic disease prophylaxis in elective hip and knee replacement surgery. Are we nearer or further away from a consensus?]. Rev Esp Cir Ortop Traumatol. 2012; 56(4):328-37. Spanish. doi: 10.1016/j.recot.2012.04.002. Epub 2012 Jun 2. PMID: 23594854.

9. AAOS. Preventing venous thromboembolic disease in patients undergoing elective hip and knee arthroplasty. 2011 [consultado 27 abril 2020], Disponible en: http://www.orthoguidelines.org/topic?id=1006.

10. Jiang Y, Du H, Liu J, Zhou Y. Aspirin combined with mechanical measures to prevent venous thromboembolism after total knee arthroplasty: a randomized controlled trial. Chin Med J (Engl) 2014; 127(12):2201-5. PMID: 24931228.

11. Hood BR, Cowen ME, Zheng HT, Hughes RE, Singal B, Hallstrom BR. Association of Aspirin With Prevention of Venous Thromboembolism in Patients After Total Knee Arthroplasty Compared With Other Anticoagulants: A Noninferiority Analysis. JAMA Surg 2019; 154(1):65-72. doi: 10.1001/jamasurg.2018.3858. PMID: 30347089; PMCID: PMC6439863.

12. Anderson DR, Morgano GP, Bennett C, et al. American Society of Hematology 2019 guidelines for management of venous thromboembolism: prevention of venous thromboembolism in surgical hospitalized patients. Blood Adv 2019; 3(23):3898-3944. doi:10.1182/bloodadvances.2019000975.

13. Jenny JY, Pabinger I, Samama CM; ESA VTE Guidelines Task Force. European guidelines on perioperative venous thromboembolism prophylaxis: Aspirin. Eur J Anaesthesiol 2018; 35(2):123-129. doi: 10.1097/EJA.0000000000000728. PMID: 29112548.

14. Park YG, Ha CW, Lee SS, Shaikh AA, Park YB. Incidence and Fate of "Symptomatic" Venous Thromboembolism After Knee Arthroplasty Without Pharmacologic Prophylaxis in an Asian Population. J Arthroplasty 2016; 31(5):1072-1077. doi:10.1016/j.arth.2015.11.028.

15. Chang MJ, Song MK, Kyung MG, Shin JH, Chang CB, Kang SB. Incidence of deep vein thrombosis before and after total knee arthroplasty without pharmacologic prophylaxis: a 128-row multidetector CT indirect venography study. BMC Musculoskelet Disord 2018; 19(1):274. doi: 10.1186/s12891-018-2166-8. PMID:30064411.

16. Blanchard J, Meuwly JY, Leyvraz PF, Miron MJ, Bounameaux H, Hoffmeyer P, Didier D, Schneider PA. Prevention of deep-vein thrombosis after total knee replacement. Randomised comparison between a lowmolecular-weight heparin (nadroparin) and mechanical prophylaxis with a foot-pump system. J Bone Joint Surg Br 1999; 81(4):654-9. doi: 10.1302/0301-620x.81b4.9464. PMID: 10463739.

17. Norgren L, Toksvig-Larsen S, Magyar G, Lindstrand A, Albrechtsson U. Prevention of deep vein thrombosis in knee arthroplasty. Preliminary results from a randomized controlled study of low molecular weight heparin vs foot pump compression. Int Angiol 1998; 17(2):93-6. PMID: 9754896.

18. Chin PL, Amin MS, Yang KY, Yeo SJ, Lo NN. Thromboembolic prophylaxis for total knee arthroplasty in Asian patients: a randomised controlled trial. J Orthop Surg (Hong Kong) 2009; 17(1):1-5. doi: 10.1177/230949900901700101. PMID: 19398783. 
19. Silbersack Y, Taute BM, Hein W, Podhaisky H. Prevention of deep-vein thrombosis after total hip and knee replacement. Low-molecular-weight heparin in combination with intermittent pneumatic compression. J Bone Joint Surg Br 2004; 86(6):809-12. doi: 10.1302/0301-620x.86b6.13958. PMID: 15330019.

20. Edwards JZ, Pulido PA, Ezzet KA, Copp SN, Walker RH, Colwell CW Jr. Portable compression device and lowmolecular-weight heparin compared with low-molecular-weight heparin for thromboprophylaxis after total joint arthroplasty. J Arthroplasty 2008; 23(8):1122-7. doi: 10.1016/j.arth.2007.11.006. Epub 2008 Apr 2. PMID: 18534421.

21. Eisele R, Kinzl L, Koelsch T. Rapid-inflation intermittent pneumatic compression for prevention of deep venous thrombosis. J Bone Joint Surg Am 2007; 89(5):1050-6. doi: 10.2106/JBJS.E.00434. PMID: 17473143.

22. Snyder MA, Sympson AN, Scheuerman CM, Gregg JL, Hussain LR. Efficacy in Deep Vein Thrombosis Prevention With Extended Mechanical Compression Device Therapy and Prophylactic Aspirin Following Total Knee Arthroplasty: A Randomized Control Trial. J Arthroplasty 2017; 32(5):1478-1482. doi:10.1016/j.arth.2016.12.027.

23. Box HN, Shahrestani S, Huo MH. Venous Thromboembolism Prophylaxis after Total Knee Arthroplasty. J Knee Surg 2018; 31(7):605-609. doi: 10.1055/s-0038-1636907. Epub 2018 Mar 7. PMID: 29514375.

24. Lynch JA, Baker PL, Polly RE, Lepse PS, Wallace BE, Roudybush D, Sund K, Lynch NM. Mechanical measures in the prophylaxis of postoperative thromboembolism in total knee arthroplasty. Clin Orthop Relat Res 1990; 260:24-9. PMID: 2225629.

25. He ML, Xiao ZM, Lei M, Li TS, Wu H, Liao J. Continuous passive motion for preventing venous thromboembolism after total knee arthroplasty. Cochrane Database Syst Rev 2014;29: 7:CD008207. doi:10.1002/14651858.CD008207.pub3. Review. PMID: 25069620.

26. Joshi RN, White PB, Murray-Weir M, Alexiades MM, Sculco TP, Ranawat AS. Prospective Randomized Trial of the Efficacy of Continuous Passive Motion Post Total Knee Arthroplasty: Experience of the Hospital for Special Surgery. J Arthroplasty 2015; 30(12):2364-2369. doi:10.1016/j.arth.2015.06.006.

27. Hui AC, Heras-Palou C, Dunn I, Triffitt PD, Crozier A, Imeson J, et al. Graded compression stockings for prevention of deep-vein thrombosis after hip and knee replacement. Journal of Bone and Joint Surgery. British Volume 1996; 78(4):550,Äê4. [PubMed] [Google Scholar]

28. Fuji T, Fujita S, Kimura T, Ibusuki K, Abe K, Tachibana S, Nakamura M. Clinical benefit of graduated compression stockings for prevention of venous thromboembolism after total knee arthroplasty: post hoc analysis of a phase 3 clinical study of edoxaban. Thromb J 2016; 14:13. doi: 10.1186/s12959-016-0087-z. PMID: 27284271 ; PMCID: PMC4899917. 